\title{
Molecular surveillance of Theileria ovis, Theileria lestoquardi and Theileria annulata infection in sheep and ixodid ticks in Iran
}

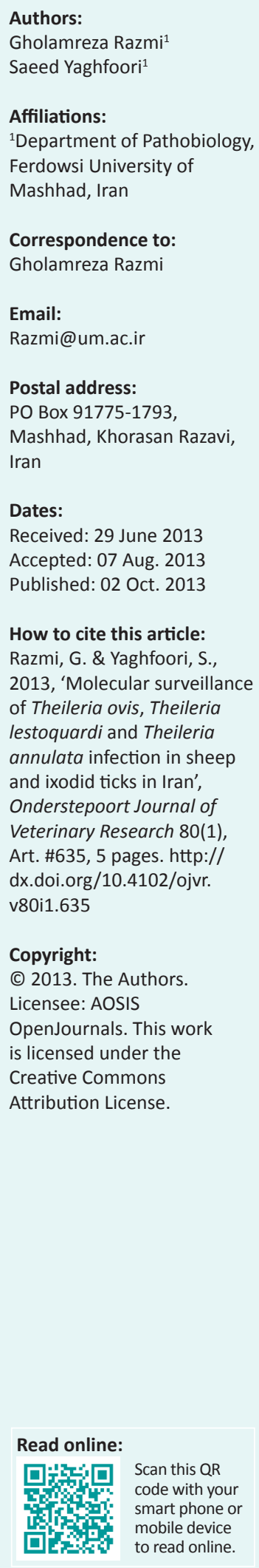

A molecular study was undertaken to detect Theileria ovis, Theileria lestoquardi and Theileria annulata in sheep and tick vectors. Investigation was conducted from 2010 to 2011 in the south of Khorasan Razavi Province, Iran. A total of 150 blood samples were collected from 30 different sheep flocks. In addition, ixodid ticks were sampled from the same flocks. The stained blood smears were microscopically examined for the presence of piroplasms and a semi-nested polymerase chain reaction-restriction (PCR) was used for subsequent molecular speciation. Salivary glands were isolated from the ticks and subsequently analysed by semi-nested PCR. polymerase chain reaction-restriction fragment length polymorphism (PCR-RFLP) was used to differentiate between T. lestoquardi and T. annulata from PCR-positive samples. Theileria species infection was microscopically detected in $18.6 \%$ of blood smears. The presence of T. ovis and T. lestoquardi or T. annulata was detected by semi-nested PCR in $58.6 \%$ and $6.6 \%$ of blood samples respectively. In total, 169 ixodid ticks were collected from different areas of the province. The most prevalent ticks were Rhipicephalus turanicus ( $n=155 ; 91.7 \%$ of the total), followed by Hyalomma anatolicum anatolicum $(n=8 ; 4.7 \%)$ and Hyalomma marginatum turanicum $(n=6 ; 3.5 \%)$. From an organ pooling of 33 ticks, three pools of salivary glands from $R$. turanicus were positive for Theileria species by semi-nested PCR. Of the three $R$. turanicus samples testing positive for Theileria species, two $(6.1 \%)$ were positive for $T$. ovis and one (3.0\%) for T. lestoquardi or T.annulata. Amongst the 11 PCR-positive samples for T. lestoquardi or T. annulata, 10 were positive for T. lestoquard $i$ and one sample was positive for both T. lestoquardi and T. annulata using PCR-RFLP. The results also demonstrated that PCR-RFLP could be used for the detection of T. ovis. Based on the results, it can be concluded that $T$. ovis has a higher prevalence than T. lestoquardi, and that $R$. turanicus could be a possible vector for T. ovis and T. lestoquardi. Finally, the PCR-RFLP based on Msp1 restriction enzyme is a simple method for differentiation of Theileria species in sheep and ixodid ticks.

\section{Introduction}

At least six Theileria species have been found to infect small ruminants. Of these, Theileria lestoquardi, Theileria luwenshuni and Theileria uilenbergi are considered to be highly pathogenic in sheep and goats. The other three species, Theileria separata, Theileria ovis and Theileria recondita, are generally considered to be non-pathogenic or mildly pathogenic (Perston 2001; Schnittger et al. 2000; Uilenberg 1995, 1997). Theileria annulata, which is a causal agent of malignant theileriosis in cattle, can also infect sheep (Leemans et al. 1999). Theileria species have been successfully identified in sheep by using different molecular methods based on the hyper-variable V4 region of $18 \mathrm{~S}$ rRNA genes. The available primers based on $18 \mathrm{~S}$ rRNA genes have also cross-reacted with T. annulata and T. lestoquardi (Gubbels et al. 1999; Schnittger et al. 2004). It is necessary to differentiate between T. lestoquardi and T. annulata because both pathogens are transmitted by Hyalomma anatolicum anatolicum and both can be found in small ruminants (Spitalska et al. 2005). Different molecular methods such as polymerase chain reaction (PCR) (Kirvar et al. 1998), polymerase chain reactionrestriction fragment length polymorphism (PCR-RFLP) (Heidarpour et al. 2009; Spitalska et al. 2005) and Lockwood Analytical Method for Prediction (LAMP) (Liu et al. 2008; Salih et al. 2008) have been developed to differentiate the two Theileria species.

Iran is an endemic region for T. ovis, T. lestoquardi and T. annulata infection in sheep and cattle. Hyalomma anatolicum anatolicum is a common tick vector for T. lestoquardi and T. annulata. To date, T. annulata infection has also been reported in sheep from Iran (Zaeemi et al. 2011). The climate in the southern part of the Khorasan Razavi province is similar to other areas in Iran where ovine theileriosis is present. In addition, many suspected cases of ovine theileriosis have been reported by local veterinary services in this area. The aim of the study is the molecular identification and differentiation of Theileria species in sheep and tick vectors from these areas. 


\section{Material and methods Field study}

The study was conducted in the southern areas of the Khorasan Razavi Province, which is located in the east of Iran (Figure 1). An approximate population of 1493838 sheep is distributed throughout this area. Sheep husbandry is one of the most economically important occupations in this area. Sheep and goat flocks are kept together and the average herd size is between 200-1000. The sheep outnumber goats and the ratio is reported to be 50-900 sheep and 30-200 goats in each flock. The climate is semi-arid, with cold winters and warm summers, and there is an average annual rainfall of $167 \mathrm{~mm}$. The mean annual temperature ranges from $5.5^{\circ} \mathrm{C}-23.0^{\circ} \mathrm{C}$.

\section{Blood samples}

Flocks with a clinical history of theileriosis in five different areas in the south of the Khorasan Razvai Province during 2010-2011 were selected for this study. Five sheep from each of these flocks were then randomly selected. Blood was drawn from the jugular vein and collected in EDTA tubes. Simultaneously, the body of each animal was inspected, and if ticks were found, they were placed into appropriately labelled vials. The blood and tick specimens were refrigerated during transport to the laboratory.

\section{Microscopy of blood smears}

Blood smears were air dried, fixed in methanol, and stained with $10 \%$ Giemsa solution in phosphate-buffered saline

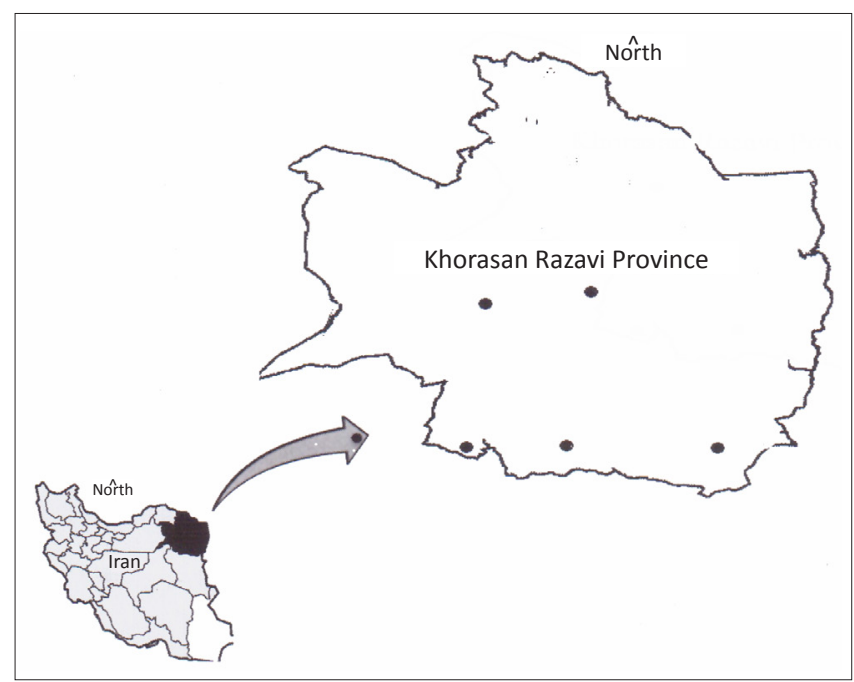

FIGURE 1: Locality where the fieldwork was carried out in Khorasan Razavi Province, Iran.
(PBS), pH 7.2. The slides were microscopically examined using an oil immersion lens at $1000 \times$ magnification.

\section{Tick examination}

The ticks collected from each infested sheep were counted and identified by microscopy using appropriate keys (Estrada-Pena et al. 2004; Hoogstraal 1956; Walker et al. 2003). The ticks were grouped into pools of five, according to their species and sex. The salivary glands from each tick pool were dissected and placed in $0.85 \%$ saline. The ticks and isolated organs were all stored at $-20{ }^{\circ} \mathrm{C}$.

\section{Semi-nested PCR}

Total genomic DNA was extracted from EDTA blood and tick samples using a DNA isolation kit (Cinna Gene, Tehran, Iran). Semi-nested PCR was performed according to the method of Shayan and Rahbari (2005).

In the first round of amplification, a forward primer (P1), 5'-CACAGGGAGGTAGTGACAAG-3', and a reverse primer (P2), 5'-AAGAATTTCACCTATGACAG-3', were used to yield an amplification product, which allowed differentiation between Theileria and Babesia. Amplification was conducted in $20 \mu \mathrm{L}$ reaction volumes (Accupower PCR PreMix kit, Bioneer, Seoul, South Korea) with a final concentration of $250 \mu \mathrm{M}$ of each dNTP in $10 \mathrm{mM}$ Tris- $\mathrm{HCl}, \mathrm{pH} 9.0,30 \mathrm{mM} \mathrm{KCl}$, $1.5 \mathrm{mM} \mathrm{MgCl2}, 1 \mathrm{U}$ Taq DNA polymerase, and $10 \mathrm{pmol}$ of each PCR primer (Takapouzist Co., Tehran, Iran). One microlitre of DNA template was added to each reaction mixture and water was added up to a total volume of $20 \mu \mathrm{L}$. The reactions were subjected to the following cycling conditions using the Bio-Rad Thermocycler: $95{ }^{\circ} \mathrm{C}$ for $5 \mathrm{~min}$, 36 cycles with denaturing at $94^{\circ} \mathrm{C}$ for $45 \mathrm{~s}$, primer annealing at $54{ }^{\circ} \mathrm{C}-58^{\circ} \mathrm{C}$ for $45 \mathrm{~s}$, and an extension step at $72{ }^{\circ} \mathrm{C}$ for $45 \mathrm{~s}$, followed by final extension at $72{ }^{\circ} \mathrm{C}$ for $10 \mathrm{~min}$. The products were refrigerated at $-4{ }^{\circ} \mathrm{C}$.

The PCR products were separated by electrophoresis on a 1.5\% agarose gel with TBE buffer and visualised using ethidium bromide and a UV-illuminator (Vilber Lourmat, Germany). Visible bands at 426 base pair (bp) - $430 \mathrm{bp}$ for Theileria species were produced in the first round of PCR. In the second round, amplification by internal primers was used to specifically detect $T$. ovis (P2/P4) and T. lestoquardi/ T. annulata (P2/P3) as shown in Table 1 . The PCR products were also separated using electrophoresis on a $1.5 \%$ agarose gel to determine the presence of specific bands for T. ovis and T. lestoquardi/T. annulata. For positive controls, T. annulata and T. lestoquardi gDNA (provided by the Razvi Vaccine and Serum Research, Karaj Branch, Karaj, Iran), and T. ovis

TABLE 1: The sequences of primers used in semi-nested polymerase chain reaction.

\begin{tabular}{llll}
\hline PCR product & Nucleotide sequences & Publication references and gene bank code & Name of Primer \\
\hline $426 \mathrm{bp}-430 \mathrm{bp}$ & 5' CACAGGGAGGTAGTGACAAG 3' & Hyper variable region V4 of 18S rRNA & 18S rRNA gene sense \\
$389 \mathrm{bp}-402 \mathrm{bp}$ & 5' AAGAATTTCACCTCTGACAG 3' & AJ006446 NCBI & 18S rRNA gene antisense \\
$235 \mathrm{bp}$ & 5' ATTGCTTGTGTCCCTCCG 3' & AF081135.1 NCBI & Thieleria lestoquardi sense \\
$237 \mathrm{bp}$ & 5' TTGCTTTTGCTCCTTTACGAG 3' & AY260171.1 NCBI & P3 \\
\hline
\end{tabular}

Source: (Schnittger et al. 2004)

PCR, polymerase chain reaction; NCBI, National center for biotechnology information; bp, base pair. 
gDNA (provided by Dr Parviz Shayan, Parasitology Dept., Faculty of Veterinary Medicine, Tehran University, Tehran, Iran) were used. A sample containing sterile water was used as a negative control and simultaneously as a control for contamination in each PCR amplification.

\section{Polymerase chain reaction-restriction fragment length polymorphism}

Theileria. annulata and T. lestoquardi were differentiated by Polymerase Chain Reaction-Restriction (PCR-RFLP) analysis of the first round of semi-nested PCR products with $M s p 1$ enzyme (MBI Fermentas, Lithuania) according to the method of Spitalska et al. (2004). This involved mixing $10 \mu \mathrm{L}$ PCR products $(0.5 \mu \mathrm{g}-1 \mu \mathrm{g}), 18 \mu \mathrm{L}$ nuclease-free water, $2 \mu \mathrm{L}$ 10× Buffer Tango, $1 \mu \mathrm{L} \mathrm{Msp1} \mathrm{in} 31 \mu \mathrm{L}$ total volume. The reaction was incubated at $37{ }^{\circ} \mathrm{C}$ for $6 \mathrm{~h}$. Five microlitres of restricted samples were transferred to a $1.5 \%$ agarose gel with a 100 bp DNA ladder (MBI Fermentas, Lithuania) for fragment size determination. DNA fragments were thereafter separated by horizontal electrophoresis in $1 \times$ TAE buffer at $80 \mathrm{~V}$ for one hour. The gel was stained using commercial ethidium bromide. The restriction analysis patterns for Theileria species are shown in Table 2. The Msp1 restriction enzyme was able to cut amplified products from $T$. ovis to distinguish it from Theileria species.

\section{Sequencing}

Selected positive products were sequenced in the facilities of Bioneer Inc. (Seoul, South Korea). Sequences were analysed

TABLE 2: Results of restriction enzyme on nucleotide sequences of different Theileria species in sheep.

\begin{tabular}{ll}
\hline Species & MSP1 enzyme \\
\hline Theileria ovis & 178.255 \\
Theileria lestoquardi & 207.222 \\
Theileria annulata & No digestion \\
\hline
\end{tabular}

by using NCBI BLAST, National Institute of Health, USA (http://www.ncbi.nlm.nih.gov).

\section{Results}

Theileria species infection was microscopically detected in $18.6 \%$ of blood smears with low parasitic infections $(0.2 \%-0.01 \%)$, whilst semi-nested PCR findings revealed that $58.6 \%(n=88)$ of blood samples were positive for T. ovis and $6.6 \%(n=10)$ for T. lestoquardi or T. annulata (Table 3$)$. The results also indicated that all positive samples from blood smears were positive when using semi-nested PCR, whereas no piroplasms were seen by microscopy in 70 PCR-positive animals. In total, 169 ixodid ticks were collected from different areas of the province. The most common tick was $R$. turanicus ( $n=155 ; 91.7 \%$ of the total). Other ticks included $H$. a. anatolicum $(n=8 ; 4.7 \%)$ and Hyalomma marginatum turanicum $(n=6 ; 3.5 \%)$. From a total of 33 tick organ pools, three pools of salivary glands from $R$. turanicus were positive for Theileria species by semi-nested PCR. Of these positive samples, two (6.1\%) were positive for T. ovis DNA and one (3\%) for T. lestoquardi or T. annulata DNA (Table 4). PCR amplification using the first primer pair produced a major product between 426 and $430 \mathrm{bp}$ for Theileria DNA. The amplified product of samples positive for T. lestoquardi or T. annulta in the first round of semi-nested PCR was further analysed by PCR-RFLP. MspI digestion gave very distinct profiles that differentiated $T$. annulata and T. lestoquardi. The results of enzymatic digestion $(n=10$, 90.9\%) by MspI showed T. lestoquardi and mixed infection $(n=1,9.1 \%)$ with $T$. annulata and T. lestoquardi (Figure 2). This method also showed that the enzyme modified is able to distinguish T. ovis (Figure 3). The sequencing results also confirmed the PCR-RFLP results. The sequenced PCR products were found to be $100 \%$ identical to the T. lestoquardi 18S rRNA GenBank references (JQ917458.1, AF081135.1, KC778786.1, KC778785.1) by blast analysis.

TABLE 3: Molecular and microscopic detection of Theileria species in blood samples of sheep in the south of the Khorasan Razavi province.

\begin{tabular}{|c|c|c|c|c|c|c|c|c|c|c|c|c|c|}
\hline \multirow[t]{2}{*}{ Area } & \multirow{2}{*}{$\begin{array}{l}\text { Number of } \\
\text { samples }\end{array}$} & \multirow{2}{*}{$\begin{array}{l}\text { Number of } \\
\text { positive } \\
\text { blood smears }\end{array}$} & \multirow[t]{2}{*}{$\%$} & \multicolumn{4}{|c|}{ Semi-nested PCR number } & \multicolumn{6}{|c|}{ RFLP-PCR number } \\
\hline & & & & $\begin{array}{l}\text { Theileria. } \\
\text { ovis }\end{array}$ & $\%$ & $\begin{array}{l}\text { Theileria lestoquardi } \\
\text { /Theileria annulata }\end{array}$ & $\%$ & $\begin{array}{l}\text { Theileria } \\
\text { lestoquardi }\end{array}$ & $\%$ & $\begin{array}{l}\text { Theileria } \\
\text { annulata }\end{array}$ & $\%$ & Mixed & $\%$ \\
\hline Ferdows & 31 & 7 & - & 23 & - & 1 & - & 1 & - & 0 & - & 0 & - \\
\hline Kashmar & 30 & 6 & - & 23 & - & 2 & - & 2 & - & 0 & - & 0 & - \\
\hline Torbat & 31 & 7 & - & 24 & - & 2 & - & 2 & - & 0 & - & 0 & - \\
\hline Kakh & 28 & 6 & - & 8 & - & 2 & - & 1 & - & 0 & - & 1 & - \\
\hline
\end{tabular}

PCR, polymerase chain reaction; PCR-RFLP, Polymerase chain reaction-restriction fragment length polymorphism; Tovis, Theileria ovis; T. lestoquardi /T. annulata, Theileria lestoquardi /Theileria annulata.

TABLE 4: Frequency of tick infestation with the results of molecular methods for detection Theileria species in salivary glands of Ixodid ticks.

\begin{tabular}{|c|c|c|c|c|c|c|c|c|c|c|c|}
\hline \multirow[t]{2}{*}{ Tick Species } & \multirow{2}{*}{$\begin{array}{l}\text { Number of } \\
\text { ticks }\end{array}$} & \multirow[t]{2}{*}{$\%$} & \multirow{2}{*}{$\begin{array}{l}\text { Tick pool } \\
\text { of salivary } \\
\text { glands }\end{array}$} & \multirow[t]{2}{*}{$\%$} & \multicolumn{4}{|c|}{ Semi-nested PCR number } & \multicolumn{3}{|c|}{ RFLP-PCR number } \\
\hline & & & & & $\begin{array}{c}\text { Theileria } \\
\text { ovis }\end{array}$ & $\%$ & $\begin{array}{c}\text { Theileria lestoquardi/ } \\
\text { Theileria annulata }\end{array}$ & $\%$ & $\begin{array}{l}\text { Theileria } \\
\text { lestoquardi }\end{array}$ & $\begin{array}{l}\text { Theileria } \\
\text { annulata }\end{array}$ & Mixed \\
\hline Rhipicephalus turanicus & 155 & 91.7 & 31 & 94.0 & 2 & - & 1 & - & 1 & 0 & 0 \\
\hline $\begin{array}{l}\text { Hyalomma anatolicum } \\
\text { anatolicum }\end{array}$ & 8 & 4.7 & 1 & 3.0 & 0 & - & 0 & - & 0 & 0 & 0 \\
\hline $\begin{array}{l}\text { Hyalomma marginatum } \\
\text { turanicum }\end{array}$ & 6 & 3.5 & 1 & 3.0 & 0 & - & 0 & - & 0 & 0 & 0 \\
\hline Total & 169 & 100 & 33 & 100 & 2 & 6.1 & 1 & 3.0 & 1 & 0 & 0 \\
\hline
\end{tabular}

PCR, polymerase chain reaction; PCR-RFLP, Polymerase chain reaction-restriction fragment length polymorphism. 


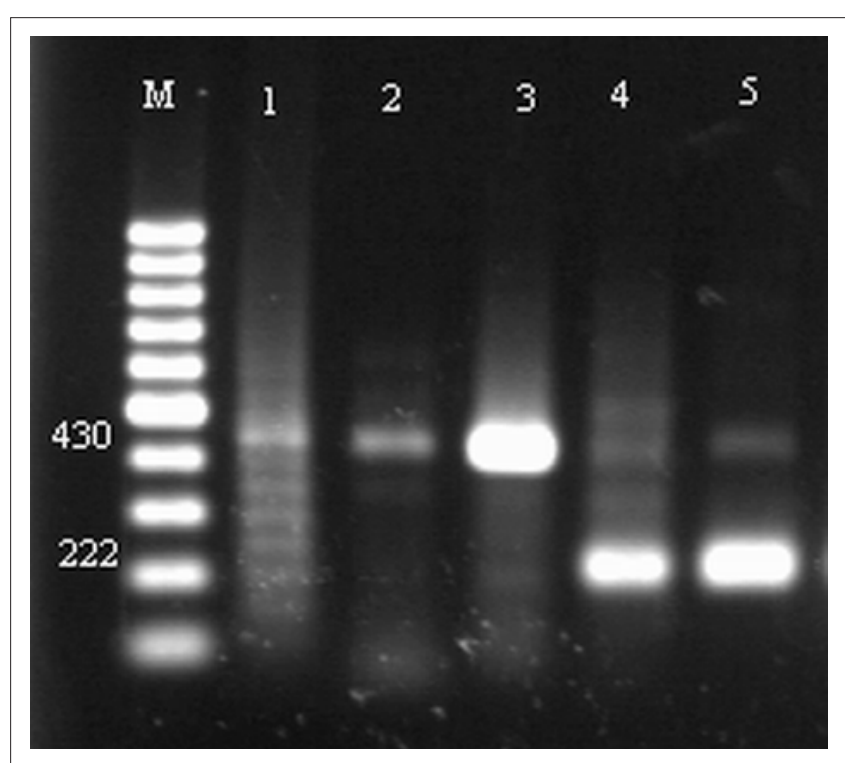

(Lane) M, DNA size marker; (Lane) 1, Theileria annulata without any enzyme; (Lane) 2, Theileria annulata Msp1 digest; (Lane) 3, Theileria lestoquardi without any enzyme; (Lane) 4, Theileria lestoquardi Msp1 digest; (Lane) 5, mixed infection Theileria lestoquardi/Theileria. annulata Msp1 digest.

FIGURE 2: Restriction digests of Theileria species amplification products.

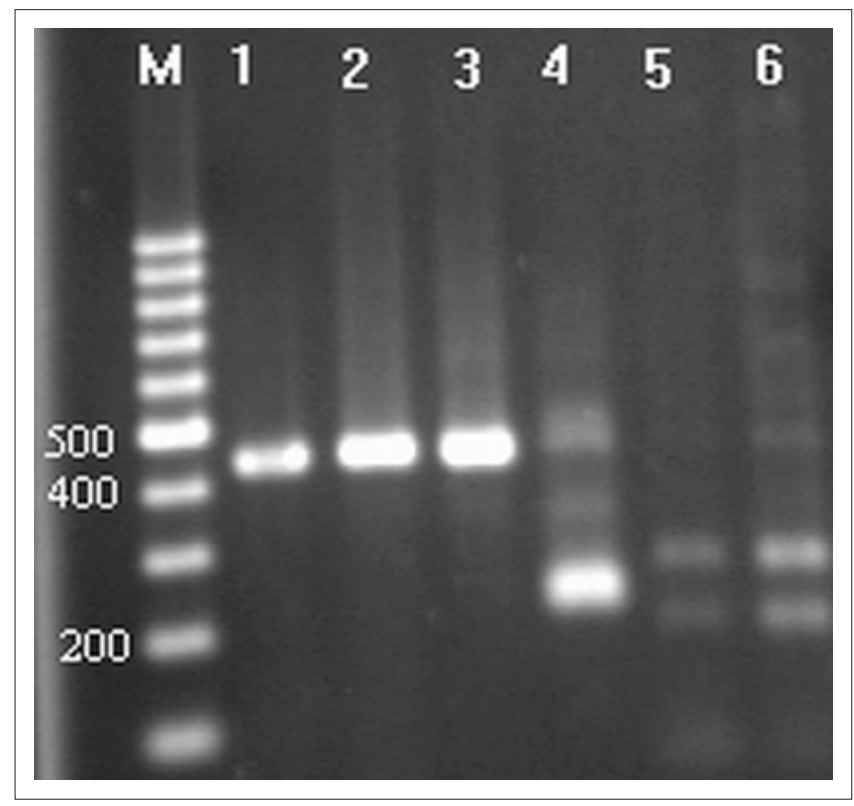

( Lane) M, DNA size marker; (Lane) 1, Theileria annulata without any enzyme; (Lane) 2 , Theileria annulata Msp1 digest; (Lane) 3, Theileria lestoquardi without any enzyme; (Lane) 4 Theileria lestoquardi Msp1 digest; (Lane) 5-6, Theileria ovis Msp1 digest.

FIGURE 3: Comparison of restriction digests on Theileria species amplification products.

\section{Discussion}

The ovine piroplasm prevalence of $18.6 \%$ detected in this study population was higher than that reported in a previous study in the southern areas of the Khorasan Razavi Province of Iran (Razmi, Eshrati \& Rashtibaf 2006). However, in contrast to previous studies, the current strategy to collect blood samples was focused on flocks with a known history of disease. In general, the infections detected in the blood smears were low and the morphological differentiation between different Theileria species was not possible. Molecular tools provide complementary diagnostic information, and accordingly, semi-nested PCR was used to achieve a simultaneous differentiation between different Theileria species in sheep (Shayan \& Rahbari 2005). With this molecular approach, $58.6 \%$ and $6.6 \%$ of blood samples were DNA-positive for T. ovis and T. lestoquardi /T. annulata, respectively.

The results demonstrated a much higher diagnostic sensitivity using PCR when compared to the conventional microscopic examination of blood smears. Our results also indicated that many of the sheep might serve as a reservoir for infection, with a very low parasitaemia that cannot be detected by microscopy.

In a previous study, Spitalska et al. (2005) detected T. lestoquardi infection in $76.0 \%$ of sheep with appropriate clinical signs in the Fars province (in southern Iran) using PCR and DNA sequencing techniques. Heidarpour et al. (2009) reported that $12.5 \%$ and $87.5 \%$ of sheep examined in the eastern half of Iran were positive for T. ovis or T. lestoquardi, respectively. Zaeemi et al. (2011) also detected T. ovis in $40.2 \%$ and T. lestoquardi in $54.8 \%$ of sheep in the western half of Iran. A comparison of these observations and this study's results indicate that the frequency of $T$. ovis infection in the Khorasan Razavi province is higher than that in any other areas of Iran, and that the T. lestoquardi infection is lower. In the present study, climatic factors may have influenced the differences in the frequency of piroplasm infection in the north when compared to the south of the province. A high prevalence of T. ovis has also been reported in some areas of Turkey (Aktas, Altay \& Dumanli 2007) and China (Ge et al. 2012).

During this study, the infected sheep did not show any clinical signs, whereas highly fatal disease due to T. lestoquardi has been reported in sheep in the south and southeast of Iran (Hashemi-Fesharaki 1997). The low incidence of clinical cases seen in the current study may be due to the low levels of infection with T. lestoquardi.

Three tick species were identified in sheep in the south Khorasan Razavi Province. Rhipicephalus turanicus was the dominant tick species in this area; it has also been reported with high prevalence in sheep from the mountainous areas such as the Khorasan province (Rahbari, Nabian \& Shayan 2007; Razmi, Najarnejad \& Rashtibaf 2011). Based on the semi-nested PCR results, T. ovis and T. lestoquradi infections were only detected in a few salivary glands in $R$. turanicus. Molecular analysis revealed that Rhipicephalus bursa and Rhipicephalus sanguineus could be a possible vector for T. ovis (Aktas, Altay \& Dumanli 2006; Telmadarraiy et al. 2012). Hyalomma anatolicum has been shown to be an important vector for T. lestoquardi in Iran and other countries (HooshmandRad \& Hawa 1973; Kirvar et al.1998; Taha \& Elhussein 2010; Uilenberg 1997). In our study, few samples of H. anatolicum were collected and Theileria species were not detected by PCR. The low frequency of $H$. anatolicum has previously been reported for sheep in the Khorasan province (Razmi et al. 2003; Razmi, Najarnejad \& Rashtibaf 2011). The primer for semi-nested PCR is derived from the hypervariable region V4 of $18 \mathrm{~S}$ rRNA, and it detects and differentiates simultaneously Theileria and Babesia in DNA samples from sheep (Shayan \& 
Rahbari 2005). However, this technique cannot differentiate between T. annulata and T. lestoquardi. The polymerase chain reaction-restriction fragment length polymorphism (PCRRFLP) can easily discriminate between T. lestoquardi and T. annulata by a single restriction digest (Spitalska et al. 2004). Furthermore, $91 \%$ of positive samples for T. lestoquardi/ T. annulata were differentiated as T. lestoquardi and $9 \%$ were found to have a mixed T. annulata and T. lestoquardi infection. This technique also shows that the method is applicable for T. ovis differentiation. Recently, different Theileria species in sheep were detected by nested PCR-RLFP (Heidarpour et al. 2009; Zaeemi et al. 2011). Naturally occurring T. annulata infection has also been reported in sheep in Iran and Sudan (Taha et al. 2013; Zaeemi et al. 2011).

\section{Conclusion}

In summary, our study indicated that microscopic examination of stained blood smears has limited value for differentiation between Theileria species, especially with low parasitaemia levels in sheep. The results of molecular examination indicated that $T$. ovis has a high prevalence when compared to T. lestoquardi in the southern Khorasan Razavi province. The dominant tick was found to be $R$. turanicus and it may act as an important vector for T. ovis and T. lestoqaurdi. Finally, PCR-RFLP based on Msp 1 restriction enzyme digestion is an applicable method for differentiation between T. lestoquardi, T. annulata and T. ovis species in sheep and tick vectors.

\section{Acknowledgements Competing interests}

The authors declare that they have no financial or personal relationship(s) that may have inappropriately influenced them in writing this article.

\section{Authors' contributions}

G.R. (Ferdowsi University of Mashhad) was the project leader and performed most of the experiments and wrote the manuscript. S.Y. (Ferdowsi University of Mashhad) was a student of Veterinary Parasitology who assisted in the examination of some experiments.

\section{References}

Aktas, M., Altay, K. \& Dumanli, N., 2006, 'PCR-based detection of Theileria ovis in Rhipicephalus bursa adult ticks', Veterinary Parasitology 140, 259-263. http:// dx.doi.org/10.1016/j.vetpar.2006.04.005, PMid:16682122

Aktas, M., Altay, K. \& Dumanli, N., 2007, 'Molecular identification, genetic diversity and distribution of Theileria and Babesia species infecting small ruminants', Veterinary Parasitology 147, 161-165. http://dx.doi.org/10.1016/j.vetpar.2007.04.001, PMid:17490817

Estrada-Peña, A., Bouattour, A., Camicas, J.L. \& Walker, A.R., 2004, Ticks of domestic animals in Mediterranean region, a guide to identification of species, published by Univerity of Zaragoza, Spain.

Ge, Y., Pan, W. \& Yin, H., 2012, 'Prevalence of Theileria infections in goats and sheep in southeastern China', Veterinary Parasitology 186, 466-469. http://dx.doi. org/10.1016/j.vetpar.2011.11.066, PMid:22178410

Gubbels, M.J., De Vos, A.P., Van der Weide, M., Viseras, J., Schouls, L.M., De Vries, E. et al. 1999, 'Simultaneous detection of bovine Theileria and Babesia species by reverse line blot hybridization', Journal of Clinical Microbiology 37, 1782-1789. PMid:10325324 PMCid:PMC84950

Hashemi-Fesharki, R., 1997, Tick-borne disease of sheep and goats and their related vectors in Iran', Parassitologia 39, 115-117. PMid:9530694
Hoogstraal, H., 1956, 'African ixodidae', in Ticks of the Sudan, Research report, vol. 1, Naval Medical Research Unit, Washington, D.C.

Hooshmand-Rad, P. \& Hawa, N.J., 1973, 'Transmission of Theileria hirci in sheep Hyalomma anatolicum anatolicum', Tropical Animal Health and Production 5, 103-109. http://dx.doi.org/10.1007/BF02236145

Heidarpour Bami, M., Haddadzadeh, H.R., Kazemi, B., Khazraiinia, P., Bandehpour, M. \& Aktas, M., 2009, 'Molecular identification of ovine Theileria species by a new PCR-RFLP method', Veterinary Parasitology 161, 171-177. http://dx.doi. org/10.1016/j.vetpar.2009.01.035, PMid:19264409

Kirvar, E., Ilhan, T., Katzer, F., Wilkie, G., Hooshmand-Rad, P. \& Brown, C.G.D., 1998 'Detection of Theileria lestoquardi (hirci) in ticks, sheep and goats using the polymerase chain reaction', Annals of the New York Academy of Sciences 29, 5262. http://dx.doi.org/10.1111/j.1749-6632.1998.tb11033.x

Leemans, I., Brown, D., Hooshmand-Rad, P., Kirvar, E. \& Uggla, A., 1999, 'Infectivity and cross-immunity studies of Theileria lestoquardi and Theileria annulata in sheep and cattle. I. In vivo responses', Veterinary Parasitology 82, 179-192. http://dx.doi.org/10.1016/S0304-4017(99)00013-8

Liu, Z., Hou, J., Bakheit, M.A., Salih, D.A., Luo, J., Yin, H. et al., 2008, 'Development of loop-mediated isothermal amplification (LAMP) assay for rapid diagnosis of ovine theileriosis in China', Parasitology Research 103, 1407-1412. http://dx.doi. org/10.1007/s00436-008-1149-3, PMid:18751728

Perston, P.M., 2001, 'Theileriosis', in M.W. Service (ed.), Encyclopedia of arthropodtransmitted infections, CAB International, Wallingford, Oxon, U.K.

Rahbari, S., Nabian, S. \& Shayan, P., 2007, 'Primary report on distribution of tick fauna in Iran', Parasitology Research 101 (suppl. 2), S175-S177. http://dx.doi. org/10.1007/s00436-007-0692-7, PMid:17823823

Razmi, G.R., Eshrati, H. \& Rashtibaf. M., 2006, 'Prevalence of Theileria spp. infection in sheep in South Khorasan province, Iran', Veterinary Parasitology 140, 239-243. http://dx.doi.org/10.1016/j.vetpar.2006.04.002, PMid:16730905

Razmi, G.R., Naghibi, A., Aslani, M.R., Dastjerdi, K. \& Hosseini, H., 2003, 'An epidemiological study on Babesia infection in small ruminants in Mashhad suburb, Province of Khorasan, Iran', Small Ruminant Research 50, 39-44. http:// suburb, Province of Khorasan, Iran', Small Rumi
dx.doi.org/10.1016/S0921-4488(03)00107-X

Razmi, G.R., Najarnejad, V. \& Rashtibaf, M., 2011, ‘Determination the frequency of Ixodid ticks on the sheep in Khorasan Razavi province, Iran', Archive Razi Institute 66, 129-132.

Salih, D.A., Liu, Z., Bakheit, M.A., Ali, A.M., El Hussein, A.M., Unger, H. et al., 2008, 'Development and evaluation of a loop-mediated isothermal amplification method for diagnosis of tropical theileriosis', Transboundary and Emerging Diseases 55, 238-43. http://dx.doi.org/10.1111/j.1865-1682.2008.01033.x, PMid:18666968

Schnittger, L., Yin, H., Jianxun, L., Ludwig, W., Shayan, P., Rahbari., S. et al., 2000 'Ribosomal small-subunit RNA gene-sequence analysis of Theileria lestoquardi and a Theileria species highly pathogenic for small ruminants in China', Parasitology Research 86, 352-358. http://dx.doi.org/10.1007/s004360050680, PMid:10836509

Schnittger, L., Yin, H., Qi, B., Gubbels, M.J., Beyer, D., Niemann, S. et al., 2004 'Simultaneous detection and differentiation of Theileria and Babesia parasites infecting small ruminants by reverse line blotting', Parasitology Research 92, 189196. http://dx.doi.org/10.1007/s00436-003-0980-9, PMid:14652747

Shayan, P. \& Rahbari. S., 2005, 'Simultaneous differentiation between Theileria spp. and Babesia spp. on stained blood smear using PCR,' Parasitology Research 97 281-286. http://dx.doi.org/10.1007/s00436-005-1434-3, PMid:16007464

Spitalska, E., Namavari, M.M., Hosseini, M.H., Shad-del, F., Amrabad, O.R. \& Sparagano. O.A.E., 2005, 'Molecular surveillance of tick-borne diseases in Iranian small ruminants', Small Ruminant Research 57, 245-248. http://dx.doi. org/10.1016/j.smallrumres.2004.07.005

Spitalska, E., Torina, A., Cannella, V., Caracappa, S. \& Sparagano, O.A., 2004, 'Discrimination between Theileria lestoquardi and Theileria annulata in their vectors and hosts by RFLP based on the 18S rRNA gene', Parasitology Research 94 , 318-20. http://dx.doi.org/10.1007/s00436-004-1217-2, PMid:15372232

Taha, K.M. \& Elhussein, A.M., 2010, 'Experimental transmission of Theileria lestoquardi by developmental stages of Hyalomma anatolicum ticks', Parasitology Research 107, 1009-1012. http://dx.doi.org/10.1007/s00436-010-1968-x, PMid:20607288

Taha, K.M., Salih, D.A., Ali, A.M, Omer, R.A. \& El Hussein, A.M., 2013, 'Naturally occurring infections of cattle with Theileria lestoquardi and sheep with Theileria annulata in the Sudan', Veterinary Parasitology 191, 143-145. http://dx.doi. org/10.1016/j.vetpar.2012.08.003, PMid:22951302

Telmadarraiy, Z., Oshaghi, M.A., Hosseini-Vasoukolaei, N., Yaghoobi-Ershadi, M.R., Babamahmoudi, F. \& Mohtarami, F., 2012, 'First molecular detection of Theilerio ovis in Rhipicephalus sanguineus ticks in Iran', Asian Pacific Journal of Tropical Medicine 5, 29-32. http://dx.doi.org/10.1016/S1995-7645(11)60240-X

Uilenberg, G., 1995, 'International collaborative research: significance of tick-borne hemoparasitic diseases on world animal health', Veterinary Parasitology 57, 1941. http://dx.doi.org/10.1016/0304-4017(94)03107-8

Uilenberg, G., 1997, 'General review of tick-borne diseases of sheep and goats worldwide', Parasitologia 39, 161-165.

Walker, A.R., Bouattour, A., Camicas, J.L., Estrada- Peña, A., Horak, I.G., Latif, A. et al., 2003, 'Ticks of domestic animals in Africa: A guide to identification of species', Bioscience Reports, Edinburgh, Scotland, U.K.

Zaeemi, M., Haddadzadeh, H.R., Khazraiinia, P., Kazemi, B. \& Bandehpour. M., 2011 Identification of different Theileria species (Theileria lestoquardi, Theileria ovis, and Theileria annulata) in naturally infected sheep using nested PCR-RFLP', Parasitology Research 108, 837-843. http://dx.doi.org/10.1007/s00436-0102119-0, PMid:20978792 